\title{
Neuromyelitis optica spectrum disorder with massive basal ganglia involvement: a case report
}

\author{
Shinji Ohara ${ }^{1,2^{*}}$, Taka-aki Miyahira ${ }^{1}$, Kenya Oguchi ${ }^{1}$, Yo-ichi Takei ${ }^{1}$, Fumihiro Yanagimura ${ }^{3}$, Izumi Kawachi ${ }^{3}$,
} Kiyomitsu Oyanagi ${ }^{4,5}$ and Akiyoshi Kakita ${ }^{6}$

\begin{abstract}
Background: Occurrence of basal ganglia involvement in neuromyelitis optica spectrum disorders (NMOSD) has rarely been reported and none documented pathologically.

Case presentation: A 73-year-old female was clinically diagnosed with a NMOSD based on the clinical and radiological features and positive serum autoantibodies to AQP4. One month before her death, she became acutely ill with disturbed consciousness and right hemiparesis, and was diagnosed and treated as having basal ganglia infarction based on the brain CT. She made a partial recovery but later died from heart failure. At autopsy, the corresponding basal ganglia process revealed a large fresh area of necrosis. Histologically, several pathological signatures of NMOSD could be recognized in the lesion, including inflammatory cell infiltrations by B and T lymphocytes, perivascular complement and fibrinogen deposition, and the appearance of numerous phagocytosed corpora amylacea within the infiltrating macrophages.

Conclusions: The present case illustrates that basal ganglia may be directly involved in the pathological processes of NMOSD, although the possibility of modification of the lesions by superimposed regional ischemia could not be excluded.
\end{abstract}

Keywords: Neuromyelitis optica spectrum disorder (NMOSD), Astrocytopathy, Basal ganglia, Blood brain barrier

\section{Background}

Neuromyelitis optica spectrum disorder (NMOSD) is a severe inflammatory autoimmune disease of the central nervous system (CNS) associated with episodes of transverse myelitis, optic neuritis and other neurologic manifestations. Antoantibodies to the water channel aquaporin-4 (AQP4), which is predominantly expressed in astrocyte foot processes, is a serum biomarker and is expressed in a majority of the cases with this syndrome [1].

Neuropathologically, NMOSD has been regarded as an autoimmune astrocytopathy, in which vasculocentric inflammatory cell inflammation and complement deposition are cardinal features [2]. This astrocytopathy results in the formation of necrotic lesions typically in the spinal cord

\footnotetext{
* Correspondence: shinji.ohara@iida.or.jp

${ }^{1}$ Department of Neurology, Matsumoto Medical Center, Minami 2-20-30, Matsumoto 399-8701, Japan

2Department of Neurology, lida Hospital, 1-15 Ohdori, lida 395-8505, Japan Full list of author information is available at the end of the article
}

and in the optic tracts, often associated with macroscopic cavity formation [3]. Other CNS areas than spinal cord and optic tracts could also be involved in NMOSD, such as the cerebral hemisphere, internal capsule, and periventricular AQP4 enriched regions including the area postrema and hypothalamus [1, 3, 4]. However, direct involvement of the deep cerebral central gray matter especially of basal ganglia has rarely been documented.

We here report a patient with NMOSD who developed a large basal ganglia lesion 1 month prior to her death, which was initially diagnosed as an ischemic infarction. However, autopsy revealed unequivocal immunohistological features of NMO in the basal ganglia lesion.

\section{Case presentation}

A previously healthy 63 year-old Japanese female presented with gait disturbance and was diagnosed as having transverse myelopathy with a sensory level of T5 and 
bilateral Babinski signs. She was given intravenous methyl prednisolone, resulting in a complete clinical recovery. At age 67, she started to complain of numbness on the left side of her face and developed an ataxic gait. MRI of the brain and the spinal cord revealed a focal lesion in the pontine tegmentum on the right, a cystic lesion in the subcortical white matter lateral to the basal ganglia on the right (Fig. 1a, b), and a longitudinally extending cavitary lesion affecting the $\mathrm{C} 2-\mathrm{C} 6$ cervical cord (Fig. 1c). Cerebrospinal fluid (CSF) examination revealed normal protein content $(43 \mathrm{mg} / \mathrm{dl})$ with increased myelin basic protein $(\mathrm{MBP})(253 \mathrm{pg} / \mathrm{ml}$, normal < 120). Oligoclonal bands were negative. Steroid therapy resulted in a good recovery. At age 69, she developed optic neuritis bilaterally. Serum antibodies to AQP4 was positive, and she was diagnosed as having NMOSD. After intravenous steroid therapy, she was started on a low maintenance dose of oral prednisone of $15 \mathrm{mg}$. At age 70, she was unable to walk and was admitted to our hospital. The neurologic examination revealed the patient had normal mental status, bilaterally decreased vision, mild dysarthria, weakness and sensory loss in the right upper extremity, and spastic paraplegia. Steroid administration was not effective in reversing her condition and she became wheelchair bound. At age 72, she developed acute myocardial infarction which was successfully treated with a stent placement. She was incidentally found to have aplastic anemia and received blood transfusions. One month prior to death, she developed right hemiparesis and disturbed consciousness over several days, and the brain CT revealed a large low-density lesion involving the left basal ganglia (Fig. 2d, e). A diagnosis of acute infarction was made and she was treated conservatively. She gradually recovered consciousness but never returned to her baseline. She died from heart failure at age 73.

The general autopsy was granted and was performed 1 hour after the death, which revealed old and recent myocardial infarcts and aspergillosis of the lung.

There was no evidence of systemic vasculitis. The brain was small and weighed $1080 \mathrm{~g}$. There were mild atheromatous changes in the basal arteries of Willis. The bilateral optic nerves and chiasma were atrophic.

On the coronal sections, there was a large soft necrotic focus involving the putamen, globus pallidus, internal capsule and caudate nucleus on the left side (Fig. 2a). A soft necrotic lesion was also seen in the corpus callosum (arrowhead in Fig. 2a). On the opposite side of the cerebral hemisphere, there were cystic lesions in the subcortical and deep white matter (Fig. 2a. Thin and thick arrows). In the brainstem, there was a focal plaque-like lesions in the medial lemniscus. The spinal cord revealed central cavitary lesions extending from C3 to C5.

Formalin-fixed, paraffin embedded sections of the brain and the spinal cord were stained with Hematoxylin
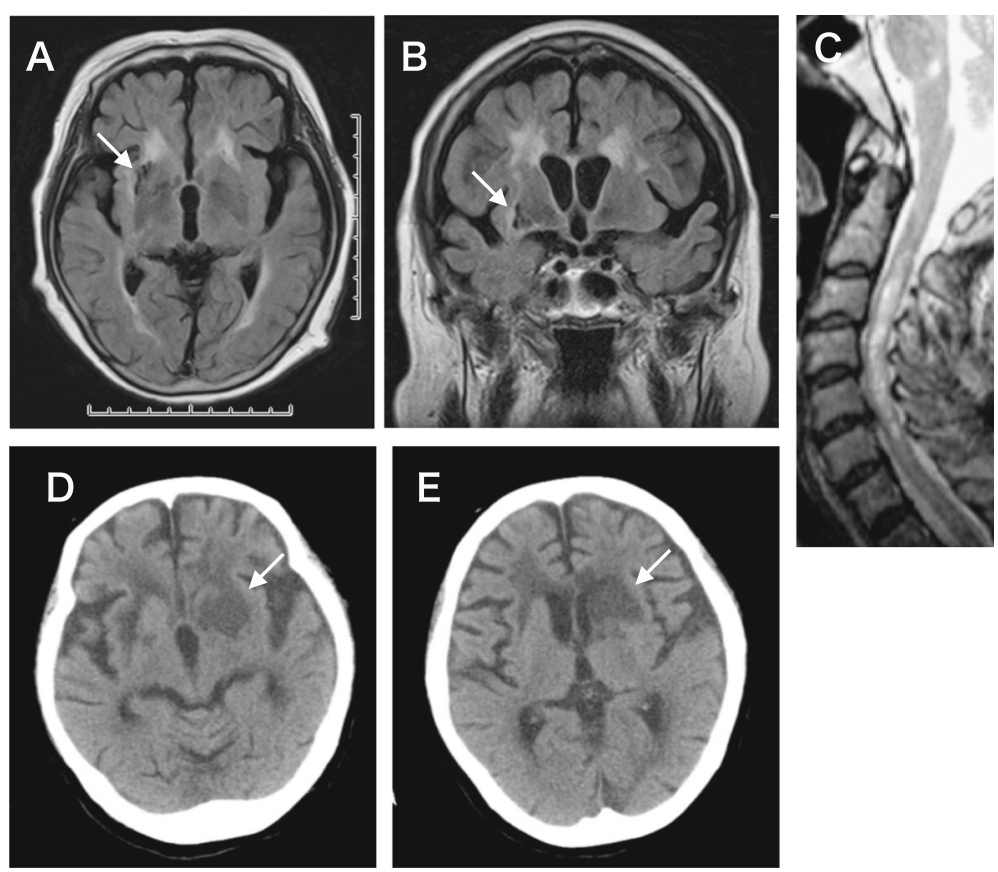

Fig. 1 Radiologic findings. a-c) Brain MRI images taken 3 years after the onset revealed a cystic lesion in the right basal ganglia (arrow), which was subsequently histologically examined at autopsy (Fig. 2a, j). a) T2 MRI, b) Flair MRI. c) T2-MRI of the spinal cord taken 4 years after the onset, revealing longitudinal lesions involving C3-6 spinal segments. d) Brain CT taken 1 month prior to the death, a few days after the patient developed right hemiparesis and disturbed consciousness. The left basal ganglia show extensive low densities accompanied by narrowing of the lateral vetricles (arrow) 

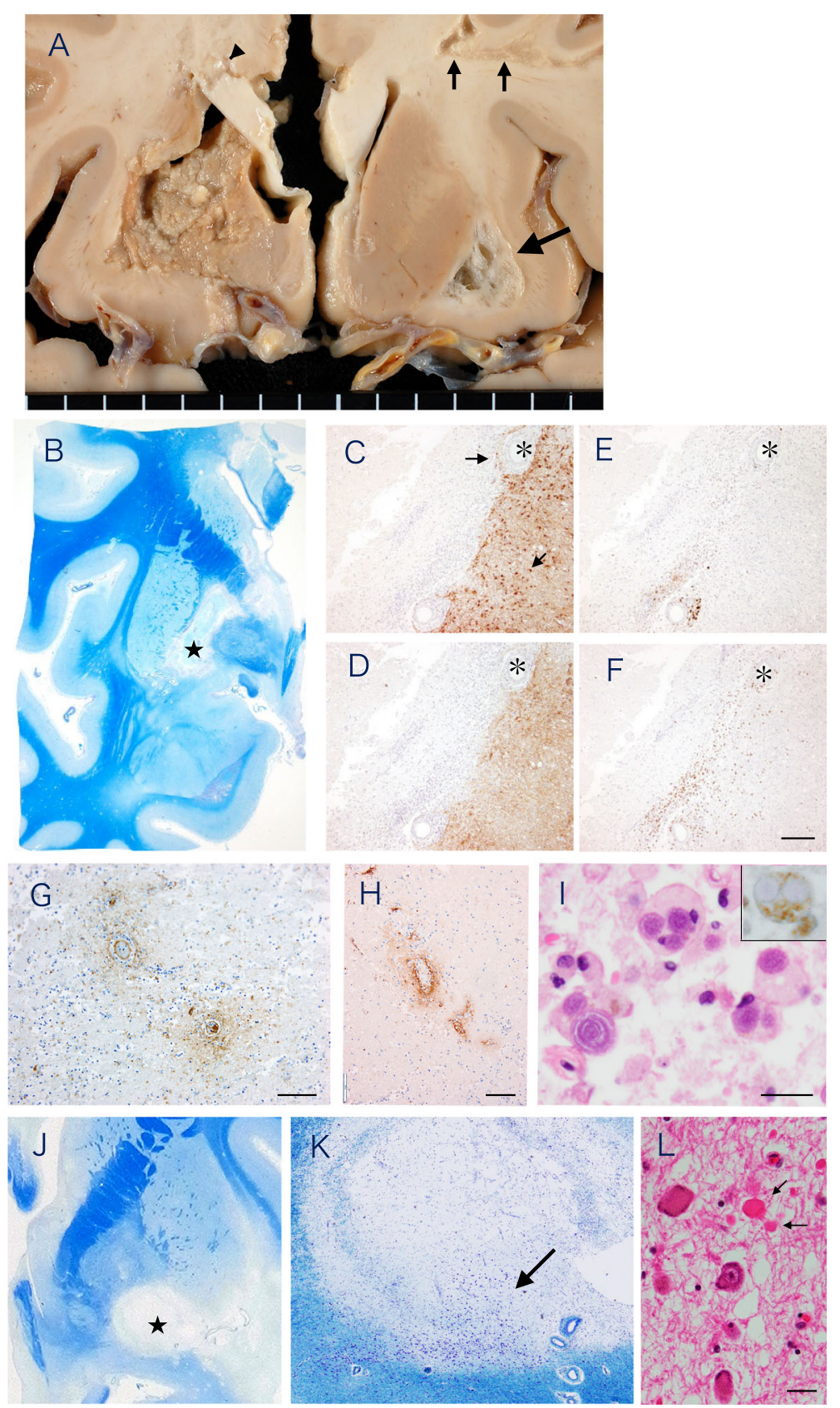

Fig. 2 (See legend on next page.) 


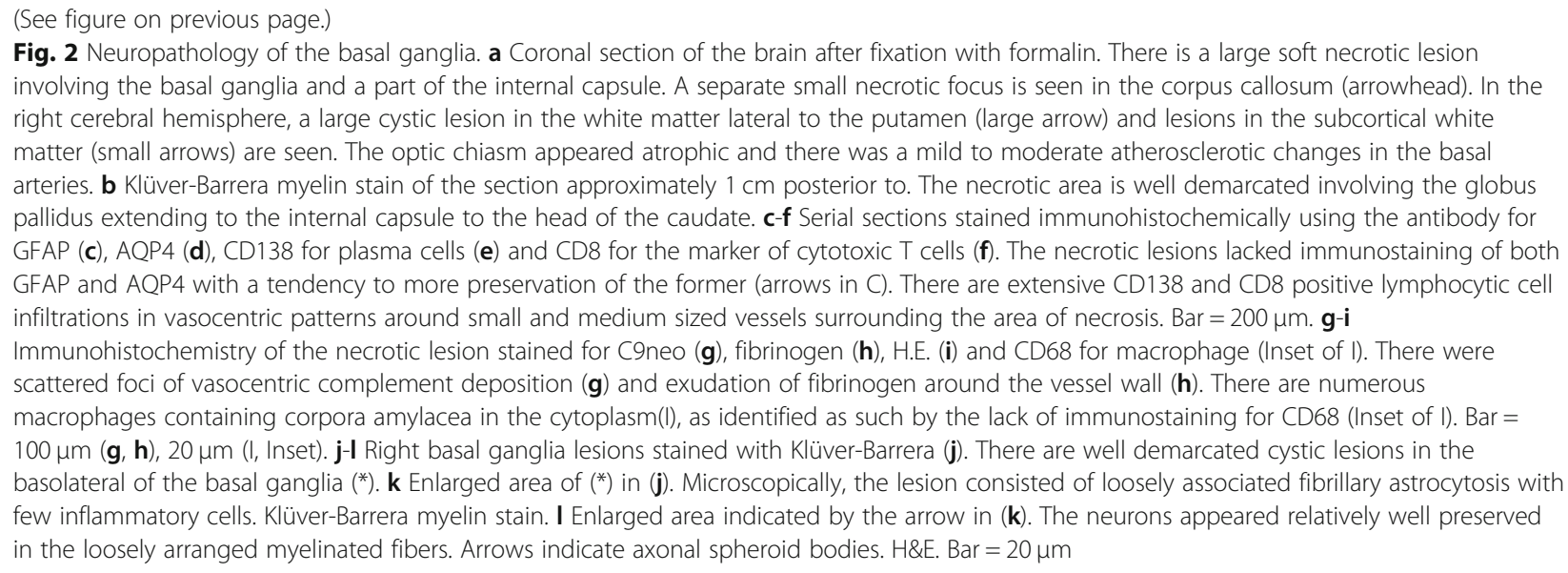

and Eosin (H\&E), Klüver-Barrera (KB) and Grocott methenamine silver staining for fungi. Immunohistochemistry with a streptavidin-Biotin method was performed with the following antibodies. Rabbit polyclonal antibodies against AQP4 (Chemicon, 1:1000), glial fibrillary acidic protein (GFAP; DAKO, 1:800), myelin basic protein (MBP 1:150, Novocastra), the cytotoxic T-cell marker CD8 (1:50, DAKO), the plasma cell marker CD138 (1:200, DAKO), macrophage/microglial marker CD68 (1:100, DAKO), fibrinogen (1:3000, DAKO), C9neo (Dr. Paul Morgan, UK, 1:200). Reaction products were visualized with DAB.

Histologically, optic nerves revealed moderate gliosis without active features of neuronal degeneration. The wall of the small vessels in the optic nerve invariably showed hyalinized thickening. The necrotic foci in the left ganglia stained pale with $H \& E$ and $K B$ stains, and was well demarcated and irregular in contour (Fig. 2b). The lesion was largely GFAP and AQP4 negative, with some tendency of relatively more GFAP preservation than AQP4 (Fig. 2c, d). At the margin surrounding the lesion, there was a prominent perivascular inflammatory cell infiltration around small and medium sized vessels. Many of the infiltrating cells were immunoreactive either for $\mathrm{B}$ and $\mathrm{T}$ lymphocyte or plasma cell markers (Fig. 2e, f). Immunohistochemistry with antibodies for C9neo and fibrinogen were positive in vasocentric patterns, which was found exclusively in the necrotic lesion (Fig. 2g, h). Numerous macrophages were also found to contain corpora amylacea in their cytoplasm (Fig. 2i). The Grocott methenamine silver stain was negative in the necrotic lesions. Occasional eosinophils and neutrophils could be recognized in the macroscopically fresh appearing lesion of the corpus callosum.

By contrast, the cystic lesions contralateral hemispheres were lined by fibrillary gliosis and there were few inflammatory cells (Fig. 2j-l). In the periphery of a lesion, many viable looking neurons were seen within the loosely arranged myelinated fibers (Fig. 2k, l). No thrombotic occlusions could be found in the lenticulostriate arteries on both sides despite an intensive search. There were no cortical demyelinating lesions except for several scattered foci of ischemic infarcts of various stage.

In the brain stem, the pontine tegmentum showed a few foci of gliosis associated with demyelination and relatively preserved axons. In the spinal cord, there were cystic lesions predominantly involving the central gray matter of the thoracic cord. There was relative sparing of axons compared to myelin in the area surrounding the cystic lesions, where the loss of AQP4 was much broader than those area with GFAP loss. In and around the cystic lesions, small vessels with thickened hyalinized walls were seen. There were only a few infiltrating $\mathrm{T}$ and $\mathrm{B}$ cells.

\section{Discussion and conclusion}

The present case could be unequivocally diagnosed as NMOSD based on typical clinical course and imaging features, and a presence of positive serum AQP4 antibody $[1,5]$, except that, one-month pre-mortem, the patient developed consciousness disturbance and hemiparesis, which was considered to be due to a large basal ganglia infarct. However, upon autopsy, the affected basal ganglia revealed immunohistological signatures of NMO, such as perivascular deposition of active complement component and extensive vasocentric inflammatory cell infiltration consisting of $\mathrm{T}$ and $\mathrm{B}$ lymphocytes and plasma cells [2], a tendency of relative preservation of GFAP compared with AQP4 in the margin of the necrotic lesions [3]. Furthermore, numerous phagocytosed corpora amylacea were observed in the necrotic lesion. Corpora amylacea are age-dependent spherical, basophilic, PAS positive inclusions that are predominantly found in the astrocytic foot processes with certain predilections including around small vessels [6], and their appearance in phagocytosed state has been regarded as a histological hallmark of astrocytic injury in 
NMO [7]. It is speculated that corpora amylacea liberated from the degenerating astrocytic foot processes around blood vessels could be engulfed by infiltrating macrophages through intact regional blood supply [7].

Occurrence of basal ganglia involvement in NMOSD has been rarely reported in the literature $[8,9]$ and none documented pathologically. Therefore, one may wonder if NMO pathology could solely be responsible for causing such large area of necrosis in the basal ganglia as seen in the present patient. In this regard, it should be noted that the presence of AQP4 antibody alone does not exhibit any pathological effects in animals and humans $[10,11]$. Nishiyama et al. suggested that certain immunologic events that increase in the Blood brain barrier (BBB) permeability are needed to allow entry and binding to AQP4 on astrocytes [11]. Recently, however, Jueneman et al. reported that in a rat stroke model, the animal infused with AQP4 antibodies suffered more vasogenic edema formation as compared to the controls, and that the size of the infarct was larger in the AQP4 antibody infused animals [12].

In the present case, exudation of fibrinogen could be demonstrated immunohistochemically around small vessels in the necrotic lesion, indicating the occurrence of $\mathrm{BBB}$ breakdown prior to the development of the necrotic lesion. The BBB anatomically consists of both the endothelial cells lining the capillary lumen and the surrounding astrocytic foot processes [13]. Moreover, it seems worthy of note that Ikuta et al. studied ultrastructural changes of astrocytes in various acute model of brain injuries including ischemia, and found that the first stage is characterized by swelling of the astrocytic foot processes surrounding the vessels and neurons, followed by the detachment of the astrocytic processes from vessel wall, suggesting the occurrence of BBB breakdown in the very acute stage of ischemia [14]. Therefore, it is possible to consider that pre-existing vasculopathy and/ or focal ischemia may have been contributory to the acceleration of AQP4 antibody-mediated astrocytopathy through leaky BBB, resulting in the formation of a large necrotic lesions in the basal ganglia.

Because the basal ganglia are a common site of ischemic stroke especially with elderly patients with preexisting vasculopathy, its involvement in NMOSD may have been under-diagnosed so far. Further clinicopathological studies are required to elucidate the frequency and the nature of basal ganglia lesions in NMOSD.

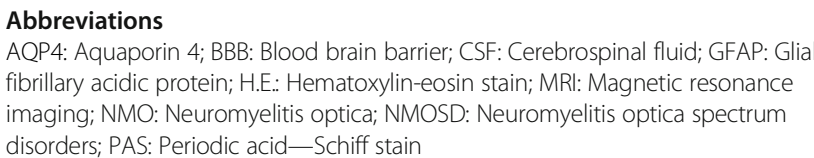

AQP4: Aquaporin 4; BBB: Blood brain barrier; CSF: Cerebrospinal fluid; GFAP: Glial fibrillary acidic protein; H.E.: Hematoxylin-eosin stain; MRI: Magnetic resonance imaging; NMO: Neuromyelitis optica; NMOSD: Neuromyelitis optica spectrum disorders; PAS: Periodic acid_-Schiff stain

\section{Acknowledgements}

The authors thank Ms. Mieko Chino, Department of Clinical Laboratory, Matsumoto Medical Center for her technical assistance. We thank Dr. B. P.
Morgan for provision of complement antibody. We also thank Dr. Takeo Misu, Department of Neurology, Tohoku University School of Medicine, Sendai, Japan, for his helpful discussion, and Prof. Robert E Schmidt, Division of Neuropathology, Washington University School of Medicine at St.Louis, for his critical reading of the manuscript.

\section{Author's contribution}

SO wrote manuscript. MT, YT, K Oguchi, SO took care of the patient and collected clinical data including CSF. TM, YT, SO and K Oyanagi performed brain cutting. FY and IK performed immunostaining of C9neo. AK performed immunostaining of AQP4 and various leucocyte antigens. SO, YT, K Oyanagi, IK and AK reviewed all of the histopathological data. All authors read and approved the manuscript.

\section{Funding}

There is no targeted funding for this publication.

\section{Availability of data and materials}

The datasets used in the current study are available form the corresponding author on reasonable request.

Ethics approval and consent to participate

Not applicable.

\section{Consent for publication}

Written informed consent was obtained from the legal guardian of the patient for publication of this autopsy case report and any accompanying images. A copy of the written consent is available for review by the Editorin-Chief of this journal.

\section{Competing interests}

The authors declare that they have no competing interests.

\section{Author details}

${ }^{1}$ Department of Neurology, Matsumoto Medical Center, Minami 2-20-30, Matsumoto 399-8701, Japan. ${ }^{2}$ Department of Neurology, lida Hospital, 1-15 Ohdori, lida 395-8505, Japan. ${ }^{3}$ Department of Neurology, Brain Research Institute, Niigata University, Niigata, Japan. ${ }^{4}$ Division of Neuropathology, Brain Research Center, Shinshu University School of Medicine, Matsumoto, Japan. ${ }^{5}$ Brain Research Laboratory, Hatsuishi Hospital, Chiba, Japan. ${ }^{6}$ Department of Neuropathology, Brain Research Institute, Niigata University, Niigata, Japan.

Received: 23 September 2019 Accepted: 19 December 2019

Published online: 30 December 2019

\section{References}

1. Wingerchuk DM, Banwell B, Benett $J$, et al. International consensus diagnostic criteria for neuromyelitis optica spectrum disorders. Neurology. 2015;85(2):177-89.

2. Lucchinetti CF, Guo Y, Popescu BFG, et al. The pathology of an autoimmune astrocytopathy: lessons learned from neuromyelitis optica. Brain Pathol. 2014;24:83-97.

3. Misu T, Hoftberger R, Fujihara K, et al. Presence of six different lesion types suggests diverse mechanisms of tissue injury in neuromyelitis optica. Acta Neuropathol. 2013:125:815-27.

4. Pittock SJ, Weinschenker BG, Luchinetti CF, et al. Neuromyelitis optica brain lesions localized at sites of high aquaporin 4 expression. Arch Neurol. 2006;63:964-8.

5. Kawachi I, Lassmann H. Neurodegeneration in multiple sclerosis and neuromyelitis optica. J Neurol Neurosurg Psychiatry. 2017:88:137.

6. Gray F, Duyckaerts C, de Gerolami U. Escourolle and Poirier's manual of basic neuropathology. 5th ed. Oxford: Oxford University Press. p. 12

7. Suzuki A, Yokoo H, Kakita A, et al. Phagocytized corpora amylacea as a histological hallmark of astrocytic injury in neuromyelitis optica. Neuropathology. 2012;32:587

8. Kim W, Park MS, Lee SH, et al. Characteristic brain magnetic resonance imaging abnormalities in central nervous system aquaporin-4 autoimmunity. Mutiple Scler. 2010;16:1229-36.

9. Matsusue E, Fiihara $Y$, Suto $Y$, et al. Three cases of neuromyelitis optica spectrum disorder. Acta Radiol Open. 2016;5(5):1-8.

10. Bradl M, Misu T, Takahashi T, et al. Neuromyelitis optica: pathogenicity of patient immunoglobulin in vivo. Ann Neurol. 2009;66:630-43. 
11. Nishiyama $\mathrm{S}$, Ito $\mathrm{T}$, Misu $\mathrm{T}$, et al. A case of $\mathrm{NMO}$ seropositive for aquaporin-4 antibody more than 10 years before onset. Neurology 72: 1960-1961, 2009 mune-mediated neurological diseases. Immunol Med. 2018;41:120-8.

12. Juenemann M, Braun T, Doenges S, et al. Aquaporin-4 autoantibodies increase vasogenic edema formation and infarct size in a rat stroke model. BMC Immunol. 2015;16:30. https://doi.org/10.1186/s12865-015-0087-y.

13. Huber VJ, Igarashi $\mathrm{H}$, Ueki S, et al. Aquaporin-4 facilitator TGN-073 promotes interstitial fluid circulation within the blood-brain barrier: [17O]H2O JJVCPE MRI study. Neuroreport. 2018;29(9):697-70.

14. Ikuta $F$. The process of brain lesion repair and activity of astrocytes. In: Ikuta F, editor. Neuropathology in brain research. Amsterdam: Excerpta Medica; 1991. p. 211-31.

\section{Publisher's Note}

Springer Nature remains neutral with regard to jurisdictional claims in published maps and institutional affiliations.

Ready to submit your research? Choose BMC and benefit from:

- fast, convenient online submission

- thorough peer review by experienced researchers in your field

- rapid publication on acceptance

- support for research data, including large and complex data types

- gold Open Access which fosters wider collaboration and increased citations

- maximum visibility for your research: over $100 \mathrm{M}$ website views per year

At BMC, research is always in progress.

Learn more biomedcentral.com/submissions 